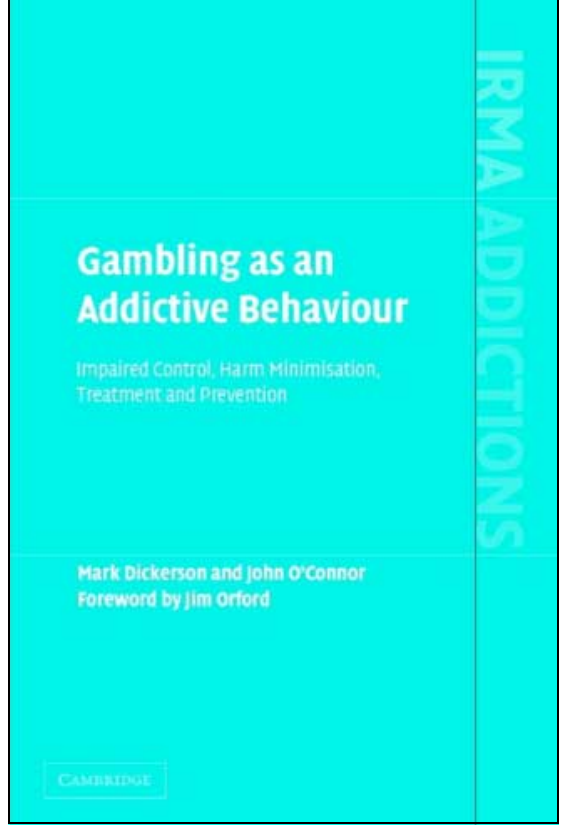

of addiction studies. It is against this background that I read this book and I found it to be timely and highly readable.

This monograph is part of the International Research Monographs in the Addictions series (the series editor is Griffith Edwards) and is authored by two experts in the field of gambling research. Although the book has an Australian bias (as the authors, the research described and the policy issues discussed are Australia-based), the theoretical constructs/models discussed and the implications for policy makers are generalisable. This book takes a look at gambling as an addictive behaviour - more specifically at the dimension of self-control over gambling behaviour and the various psychological variables that influence it. The core theme of this book is 'impaired self-control' and the authors eloquently summarise key findings from their 5-year gambling research programme. This book consists of eight chapters - all self-contained and well-organised. The authors provide an excellent overview of the key psychological variables that determine selfcontrol over gambling: emotional factors, individual differences, cognitive variables and coping, and they set this in the context of different models of impaired control and two studies that explored this subject. In addition the following topics are also well covered: implications for psychological treatment of pathological gamblers, and the concept of harm minimisation or 'responsible gambling'. I found the chapter presenting a case study of the implementation of harm minimisation strategies in
Victoria, Australia to be particularly fascinating, because of its relevance to clinicians and policy makers in the UK. This book provides interesting insights into the dimension of impaired self-control, and succeeds in highlighting its key role in the psychological conceptualisation of gambling and addictive behaviours in general.

All in all, a good read, although some of the theoretical debate presented could be intellectually taxing. At a price of $£ 55$, it may be a bit over-priced, and it may also not appeal to the non-specialist. Despite the above-noted criticisms, this book is likely to be of value to those with an interest in gambling research and policy.

Sanju George Locum consultant in addiction psychiatry, Birmingham and Solihull Mental Health Trust, B37 7UR. Email: sanju.george@talk21.com doi: 10.1192/bjp.bp.106.026187

\section{The Psychiatry of Intellectual Disability}

Edited by Ashok Roy, Meera Roy \& David Clarke. Radcliffe Publishing. 2006. 208pp. £24.95 (pb). ISBN 1857756959

Few books have been published on the psychiatry of intellectual disabilities. This book is more succinct, better presented and more consistent in the quality of writing and information than the corresponding title in the College Seminars series.

Despite this overall recommendation, the book has several flaws. It is presumably aimed primarily at trainees in the subspecialty. Clearly not a reference book, it should have been a more practical manual. Luty \& Cooper's chapter on older people with intellectual disabilities gives useful guidelines for assessment and management but other chapters are much less practiceorientated. Although certainly better than many previous texts in limiting the content regarding general 'handicap', there are still too many references to the primary healthcare of people with intellectual disabilities. The era is long gone where psychiatrists in intellectual disability act as pseudo-general practitioners. It was also unnecessary to have paragraphs on such obscure conditions as Coffin-Siris syndrome. The reality is that most referrals to psychiatrists in intellectual disabilities are for problem behaviours. The trainee must learn that it is not their responsibility to solve these problems with medication alone but they should act as the only professional who has the training and expertise to take the holistic overview of the patient in biopsychosocial terms. There is also uncritical acceptance of the vague, catch-all term of 'challenging behaviour', which hampers rather than helps approaches to problem behaviours. It was a mistake therefore to include a chapter on medication without one on basic psychological assessments and interventions that a trainee needs to understand and implement.

Roy's chapter on multidisciplinary working gives an unjustifiably rosy view of the current state of (dis)organisation of services. There are undoubtedly good working relationships between hard-working and committed professionals in learning disability services but good intentions do not compensate for lack of focused working. It is scandalous that, 15 years after its introduction, the care programme approach (CPA) has not been implemented nationwide for people with intellectual disability and mental health problems. For those who seek to improve the rights of people with intellectual disability one powerful starting point would be to demand that such people with mental health problems should have their care coordinated through the recognised national standard of the CPA. It is not good enough therefore that CPA is described in this book as 'useful' rather than 'mandatory'. Community learning disability teams, which vary in focus and make-up throughout the UK, are also blithely described as 'useful' without any recourse to evidence of service delivery models.

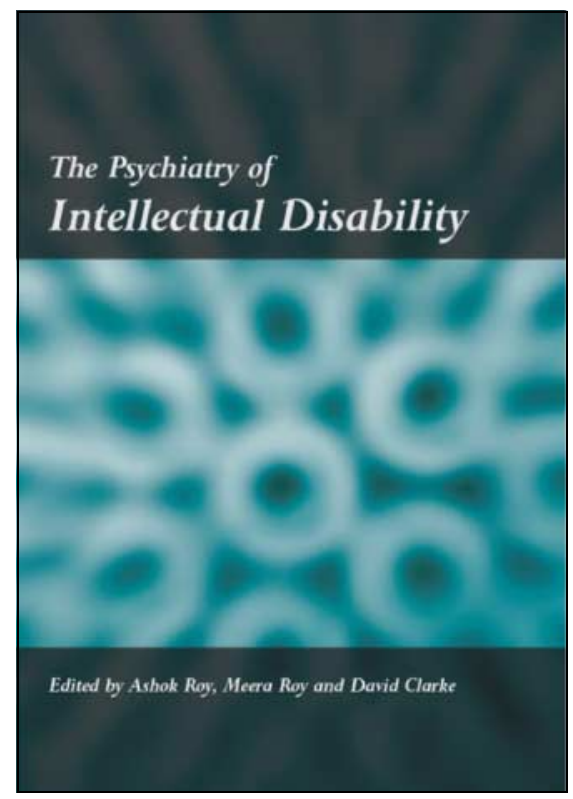


In summary, although this is perhaps the best introductory short text available in this sub-specialty, it will be unlikely to improve mental healthcare services and service delivery for people with intellectual disability.

Colin Hemmings Department of Mental Health in Learning Disabilities, South London and Maudsley NHS Trust, York Clinic, Guy's Hospital, 47 Weston Street, London SEI 3RR, UK Email: colin.hemmings@slam.nhs.uk doi: 10.I192/bjp.bp.106.026203

\section{Attachment from Infancy to Adulthood:}

\section{The Major Longitudinal Studies}

Edited by Klaus E. Grossmann

Karin Grossmann \& Everett Waters.

Guilford Press. 2005. 332pp. $€ 30.00$ (hb). ISBN I59385I456

Tracing the historical roots of attachment theory though its evolutionary stages, Attachment from Infancy to Adulthood brings the reader up to date with recent developments in the area as well as providing thoughts for the way forward into the future. It benefits greatly from contributions by people whose names are instantly recognisable for their seminal work within the area of attachment theory and it is interesting to learn about their diverse backgrounds and what motivated their interest in the subject. It is perhaps this eclectic mix of experience, and the synthesis of key disciplines including ethology, behavioural psychology and linguistics, that have enabled attachment theory to progress and develop to the extent it has over the years.

In addition to reporting findings from major longitudinal studies carried out in America and Europe, the authors discuss the many and complex methodological issues inherent in this type of research, particularly in relation to studying development across the lifespan. They examine the effects of mediating variables and their influence on the relationship between infant attachment and adult outcomes. The studies also show that it is not only mothers but fathers and, as discussed in chapter 7 , multiple caregivers in the Kibbutz setting in Israel who influence and contribute to attachment outcomes in later life. Another important dimension, as outlined in chapter 11 , is a focus on children who experience

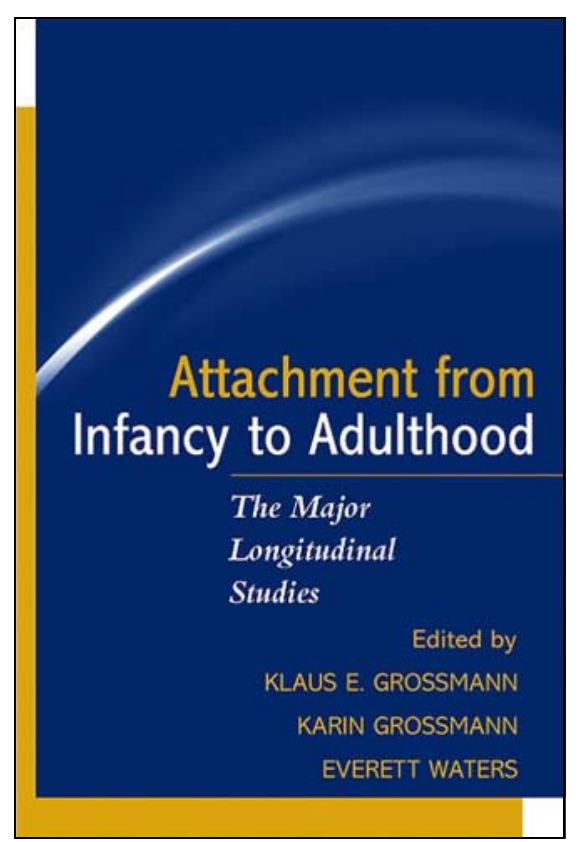

disruptions as a result of their placement in foster care.

The book is accessible and is written in an approachable manner that will appeal to students, researchers and others at various stages in their careers. One criticism I have is that the extremely naïve reader has to wait until chapter 10 before a full description of the 'strange situation' experiment, referred to throughout the book, is given.

As a proponent of longitudinal research and a user of the recently introduced Northern Ireland Household Panel survey, I found this book interesting and informative about the relationship between early attachment processes and outcomes in later life. As a parent it has caused me to study, somewhat warily, my own adult children's attachment behaviours!

Katrina L. Lloyd Queen's University Belfast, Institute of Governance, Belfast BT7 INN, UK Email: k.lloyd@qub.ac.uk

\section{doi: I0.II92/bjp.bp.106.024349}

\section{Prevention and Treatment of Suicidal Behaviour: From Science to Practice}

Edited by Keith Hawton. Oxford University Press. 2005.400 pp.US\$69.50 (pb).

ISBN 0198529767

Prevention and Treatment of Suicidal Behaviour is an accessible book that will appeal to those working in the field and those with a more casual interest. From the perspective of a trainee in psychiatry, it also clarifies the rationale behind the themes of the National Suicide Prevention Strategy for England (Department of Health, 2002). The editor, Keith Hawton, has drawn on the expertise of international authors; chapter by chapter they describe and critically appraise the evidence base, offer practical clinical guidance and identify areas in need of research.

Strategies for dealing with high-risk groups are addressed through the identification of risk factors for suicidal behaviour and chapters focusing on mental health service users, prisoners, and those who misuse substances. Qualitative research is used to identify population-based strategies for reducing suicide, which might also represent logical steps towards tackling mental illness in general. Other chapters explore the population-targeted interventions of restricted access to the means of suicide, the influence of the media's reporting, and controversies surrounding antidepressant use and suicide rates.

In addition to the two-pronged epidemiological approach to the level of intervention, the authors also present a biopsychosocial exploration of suicide prevention strategies. Psychologically, the 'entrapment model' and the role that traumatic stress has in suicidal behaviour are emphasised. Biologically, the focus shifts to descriptions of neurobiological and genetic aspects of the predisposition to suicidal behaviour. The growing concerns about self-harm and psychosocial interventions intended to reduce repetition

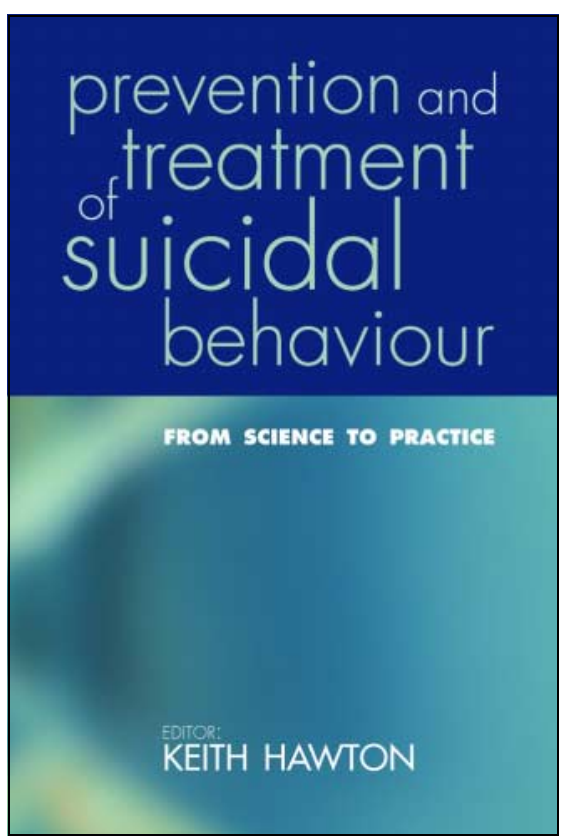

